\title{
The Wigner-Dyson-Gaudin-Mehta Conjecture
}

\author{
by Horng-Tzer Yau*
}

\author{
Department of Mathematics, Harvard University
}

\begin{abstract}
"Perhaps I am now too courageous when I try to guess the distribution of the distances between successive levels (of energies of heavy nuclei). Theoretically, the situation is quite simple if one attacks the problem in a simpleminded fashion. The question is simply what are the distances of the characteristic values of a symmetric matrix with random coefficients."
\end{abstract}

Eugene Wigner on the Wigner surmise, 1956

What are the eigenvalue statistics of a typical large matrix? Although random matrices already appeared in a concrete statistical application by Wishart in 1928 [36], these natural questions were not raised until the pioneering work [35] of E. Wigner in the 1950s. Wigner's motivation was to find a phenomenological model for the energy gap statistics of large atomic nuclei since the energy levels of large quantum systems are impossible to compute from first principles. The model Wigner proposed is the random matrix.

Consider either real symmetric or complex Hermitian matrices so that the eigenvalues are real. For definiteness, we consider $N \times N$ square matrices $H=H^{(N)}=\left(h_{i j}\right)$ with matrix elements having mean zero and variance $1 / N$, i.e.,

$$
\mathbb{E} h_{i j}=0, \quad \mathbb{E}\left|h_{i j}\right|^{2}=\frac{1}{N} \quad i, j=1,2, \ldots, N .
$$

The random variables $h_{i j}, i, j=1, \ldots, N$ are real or complex independent random variables subject to the symmetry constraint $h_{i j}=\bar{h}_{j i}$. These ensembles of random matrices are called Wigner matrices. We will always consider the limit as the matrix size goes to infinity, i.e., $N \rightarrow \infty$.

The first rigorous result about the spectrum of a random matrix of this type is the famous Wigner semicircle law [35], which states that the empirical densities of the eigenvalues, $\lambda_{1}, \lambda_{2}, \ldots, \lambda_{N}$, of large symmetric or Hermitian matrices after proper normalization such as (1) are given by

$$
\rho_{N}(x):=\frac{1}{N} \sum_{j=1}^{N} \delta\left(x-\lambda_{j}\right) \rightarrow \rho_{s c}(x):=\frac{1}{2 \pi} \sqrt{\left(4-x^{2}\right)_{+}}
$$

in the weak limit as $N \rightarrow \infty$. The limit density is independent of the details of the distribution of $h_{i j}$. Wigner further predicted that the eigenvalue gap distribution in the bulk of the spectrum is given by the Wigner surmise, e.g. in the case of symmetric matrices,

$$
\mathbb{P}\left(\frac{s}{N \rho} \leq \lambda_{j}-\lambda_{j-1} \leq \frac{s+\mathrm{d} s}{N \rho}\right) \approx \frac{\pi s}{2} \exp \left(-\frac{\pi}{4} s^{2}\right) \mathrm{d} s,
$$

" Partially supported by NSF grants DMS-0757425, 0804279 where $\rho$ is the local density of eigenvalues (see [26] for an overview).

Wigner's proof of the semicircle law was a moment method via computing $\mathbb{E} \operatorname{Tr} H^{n}$ for each $n$. The Wigner surmise proved far more challenging and difficult to comprehend. Gaudin [22] computed the specific gap distributions of random with Gaussian distribution for matrix elements in terms of a Fredholm determinant involving Hermite polynomials. Mehta and Gaudin had earlier introduced Hermite polynomials in the context of random matrices [28]. Later, Dyson and Mehta [27, 12, 13] applied this exact calculation to correlation functions and to other symmetry classes. Let $p_{N}\left(\lambda_{1}, \lambda_{2}, \ldots, \lambda_{N}\right)$ denote the joint probability density of the (unordered) eigenvalues for $N \times N$ Hermitian matrices. Then the $n$-point correlation functions (marginals) are defined by

$$
\begin{aligned}
& p_{N}^{(n)}\left(\lambda_{1}, \lambda_{2}, \ldots, \lambda_{n}\right) \\
& \quad:=\int_{\mathbb{R}^{N-n}} p_{N}\left(\lambda_{1}, \ldots, \lambda_{n}, \lambda_{n+1}, \ldots \lambda_{N}\right) \mathrm{d} \lambda_{n+1} \ldots \mathrm{d} \lambda_{N} .
\end{aligned}
$$

In the Gaussian case, the joint probability density of the eigenvalues can be expressed explicitly as

(4) $p_{N}\left(\lambda_{1}, \lambda_{2}, \ldots, \lambda_{N}\right)=$ const. $\prod_{i<j}\left(\lambda_{i}-\lambda_{j}\right)^{2} \prod_{j=1}^{N} e^{-\frac{1}{2} N \sum_{j=1}^{N} \lambda_{j}^{2}}$.

The Vandermonde determinant structure allows one to compute the $k$-point correlation functions in the large $N$ limit via Hermite polynomials that are the orthogonal polynomials with respect to the Gaussian weight function.

The final result of Dyson, Gaudin and Mehta's work can be summarized as follows: For any fixed energy $E$ in the bulk of the spectrum, i.e., $|E|<2$, the small scale behavior of $p_{N}^{(n)}$ is given explicitly by

(5)

$$
\begin{aligned}
& \frac{1}{\left[\rho_{s c}(E)\right]^{n}} p_{N}^{(n)}\left(E+\frac{\alpha_{1}}{N \rho_{s c}(E)}, E+\frac{\alpha_{2}}{N \rho_{s c}(E)}, \ldots, E+\frac{\alpha_{n}}{N \rho_{s c}(E)}\right) \\
& \quad \rightarrow \operatorname{det}\left(K\left(\alpha_{i}-\alpha_{j}\right)\right)_{i, j=1}^{n}
\end{aligned}
$$

where $K$ is the celebrated sine kernel

$$
K(x, y)=\frac{\sin \pi(x-y)}{\pi(x-y)} .
$$

The limit in (5) is independent of the energy $E$ as long as it is in the bulk of the spectrum. The rescaling by a factor $N^{-1}$ in (5) corresponds to the typical distance between 
consecutive eigenvalues. Formulas for symmetric matrices that were similar but far more complex were also obtained. It is well-known that the eigenvalue gap distribution can be computed from the correlation functions via the inclusion-exclusion principle and thus (5) also yields a precise asymptotics for eigenvalue gap distributions. In a striking coincidence, the Wigner surmise, which was based on a $2 \times 2$ matrix computation, agrees with this sophisticated formula with a typical error of only a few percentage points. Observe that the correlation functions do not factorize, i.e. despite the independent nature of the elements, the eigenvalues are strongly correlated.

Wigner envisioned that the eigenvalue gap distributions for large complicated quantum systems are universal in the sense that they depend only on the symmetry class of the physical system but not on other detailed structures. For any truly interacting system, Wigner's thesis remains unproved. Furthermore, there is no heuristically convincing case for its correctness. Nevertheless, there is a general belief that the random matrix statistics and Poisson statistics represent two paradigms of energy level statistics for many-body quantum systems: Poisson for independent systems and random matrix for highly correlated systems. In fact, these paradigms extend even to certain one-body systems such as the quantization of the geodesic flow in a domain or on a manifold [2, 4] or random Schrödinger operators [32].

The simplest class to test Wigner's universality hypothesis upon is the random matrix ensemble itself. All calculations by Dyson, Gaudin, and Mehta are for Gaussian ensembles, i.e., where the matrix elements $h_{i j}$ are real or complex Gaussian random variables. These ensembles are called the Gaussian orthogonal ensemble (GOE) and Gaussian unitary ensemble (GUE). If Wigner's universality hypothesis is correct, then the local eigenvalue statistics should be independent of the law of the matrix elements. This is generally referred to as the universality conjecture of random matrices and we will call it the Wigner-DysonGaudin-Mehta conjecture, in tribute to Wigner's vision and the pioneering work of these authors. First articulated in Mehta's treatise on random matrices [26] in 1967, it has remained a question of critical importance and concern in the subject ever since. Our goal in this paper is to review the recent progress in this direction and sketch some of the important ideas.

The laws of random matrices are generally divided into invariant and non-invariant ensembles. The invariant ensembles are characterized by a probability measure of the form $Z^{-1} e^{-N \beta \operatorname{Tr} V(H) / 2} \mathrm{~d} H$ where $N$ is the size of the matrix, $V$ is a real valued potential, and $Z$ is the normalization constant. The parameter $\beta>0$ is determined by the symmetry class of the model and $\mathrm{d} H$ is the Lebesgue measure on matrices in the class. These ensembles are called invariant since the probability law depends only on the trace of a function of the matrix and thus is invariant under changes of coordinates. The matrix elements are in general correlated and they are independent only if the model is Gaussian, i.e., $V$ is quadratic.
For invariant ensembles, the probability distribution of the eigenvalues $\lambda=\left(\lambda_{1}, \ldots, \lambda_{N}\right)$ with $\lambda_{1} \leq \ldots \leq \lambda_{N}$ for the measure $e^{-N \beta \operatorname{Tr} V(H) / 2} / Z$ is given by the explicit formula (c.f. (4))

$$
\begin{aligned}
& \mu_{\beta, V}^{(N)}(\lambda) \mathrm{d} \lambda \sim e^{-\beta N \mathscr{H}(\lambda)} \mathrm{d} \lambda \quad \text { with Hamiltonian } \\
& \mathscr{H}(\lambda):=\sum_{k=1}^{N} \frac{1}{2} V\left(\lambda_{k}\right)-\frac{1}{N} \sum_{1 \leq i<j \leq N} \log \left(\lambda_{j}-\lambda_{i}\right),
\end{aligned}
$$

where the parameter $\beta$ is determined by the symmetry class: $\beta=1$ for symmetric matrices, $\beta=2$ for Hermitian matrices and $\beta=4$ for self dual quaternion matrices. The key structural ingredient of this formula, the Vandermonde determinant, is the same as in the Gaussian case, (4). Thus all previous computations, developed for the Gaussian case, can be carried out for $\beta=1,2,4$ provided that the Gaussian weight function for the orthogonal polynomials is replaced with the function $e^{-\beta V(x) / 2}$. The asymptotic properties of the corresponding orthogonal polynomials are therefore critical to the successful analysis of the correlation functions. In the pioneering work of Dyson, Gaudin, and Mehta, the potential is the quadratic polynomial $V(x)=x^{2} / 2$ and the orthogonal polynomials are the Hermite polynomials whose asymptotic properties are well-known. For general $V$, this is a demanding task and we will review the progress after we state the main results.

Theorem 1 (Wigner-Dyson-Gaudin-Mehta conjecture). [14, Theorem 7.2] Suppose that $H=\left(h_{i j}\right)$ is a Hermitian (respectively, symmetric) Wigner matrix. Suppose that for some $\varepsilon>0$

$$
\mathbb{E}\left|\sqrt{N} h_{i j}\right|^{4+\varepsilon} \leq C
$$

for some constant $C$. Let $n \in \mathbb{N}$ and $O: \mathbb{R}^{n} \rightarrow \mathbb{R}$ be compactly supported and continuous. Let $E$ satisfy $-2<E<2$ and let $\xi>0$. Then for any sequence $b_{N}$ satisfying $N^{-1+\xi} \leq b_{N} \leq$ ||$E|-2| / 2$ we have

(9)

$$
\begin{aligned}
& \lim _{N \rightarrow \infty} \int_{E-b_{N}}^{E+b_{N}} \frac{\mathrm{d} E^{\prime}}{2 b_{N}} \int_{\mathbb{R}^{n}} \mathrm{~d} \alpha_{1} \cdots \mathrm{d} \alpha_{n} O\left(\alpha_{1}, \ldots, \alpha_{n}\right) \frac{1}{\rho_{s c}(E)^{n}} \\
& \quad \times\left(p_{N}^{(n)}-p_{\mathrm{G}, N}^{(n)}\right)\left(E^{\prime}+\frac{\alpha_{1}}{N \rho_{s c}(E)}, \ldots, E^{\prime}+\frac{\alpha_{n}}{N \rho_{s c}(E)}\right)=0 .
\end{aligned}
$$

Here $\rho_{s c}$ is the semicircle law defined in (2), $p_{N}^{(n)}$ is the $n$-point correlation function of the eigenvalue distribution of $H$, and $p_{\mathrm{G}, N}^{(n)}$ is the n-point correlation function of an $N \times N$ GUE (respectively, GOE) matrix.

In the case of invariant ensembles, it is well-known that for $V$ satisfying certain mild conditions the sequence of one-point correlation functions, or densities, associated with $\mu^{(N)}$ has a limit of $N \rightarrow \infty$ and the limiting equilibrium density $\rho(s)$ can be obtained as the unique minimizer of the functional

$$
I(v)=\int_{\mathbb{R}} V(t) v(t) \mathrm{d} t-\int_{\mathbb{R}} \int_{\mathbb{R}} \log |t-s| v(s) v(t) \mathrm{d} t \mathrm{~d} s .
$$


Moreover, for convex $V$ the support of $\rho$ is a single interval $[A, B]$ and $\rho$ satisfies the equation

$$
\frac{1}{2} V^{\prime}(t)=\int_{\mathbb{R}} \frac{\rho(s) \mathrm{d} s}{t-s}
$$

for any $t \in(A, B)$. For the Gaussian case, $V(x)=x^{2} / 2$, the equilibrium density is given by the semicircle law $\rho=\rho_{s c}$, see (2).

Theorem 2 (Bulk universality of $\beta$-ensemble). [5, 6] Assume $V$ is a real analytic function with $\inf _{x \in \mathbb{R}} V^{\prime \prime}(x)>-C$. Let $\beta>0$. Consider the $\beta$-ensemble $\mu=\mu_{\beta, V}^{(N)}$ given in (7) and let $p_{N}^{(n)}$ denote the n-point correlation functions of $\mu$, defined analogously to (3). For the Gaussian case, $V(x)=x^{2} / 2$, the correlation functions are denoted by $p_{G, N}^{(n)}$. Let $E \in(A, B)$ lie in the interior of the support of $\rho$ and similarly let $E^{\prime} \in(-2,2)$ be inside the support of $\rho_{s c}$. Let $O: \mathbb{R}^{n} \rightarrow \mathbb{R}$ be a smooth, compactly supported function. Then for $b_{N}=$ $N^{-1+\xi}$ with any $0<\xi \leq 1 / 2$ we have

$$
\begin{aligned}
& \lim _{N \rightarrow \infty} \int \mathrm{d} \alpha_{1} \cdots \mathrm{d} \alpha_{n} O\left(\alpha_{1}, \ldots, \alpha_{n}\right) \\
& \times\left[\int_{E-b_{N}}^{E+b_{N}} \frac{\mathrm{d} x}{2 b_{N}} \frac{1}{\rho(E)^{n}} p_{N}^{(n)}\left(x+\frac{\alpha_{1}}{N \rho(E)}, \ldots, x+\frac{\alpha_{n}}{N \rho(E)}\right)\right. \\
& \left.-\int_{E^{\prime}-b_{N}}^{E^{\prime}+b_{N}} \frac{\mathrm{d} x}{2 b_{N}} \frac{1}{\rho_{s c}\left(E^{\prime}\right)^{n}} p_{\mathrm{G}, N}^{(n)}\left(x+\frac{\alpha_{1}}{N \rho_{s c}\left(E^{\prime}\right)}, \ldots, x+\frac{\alpha_{n}}{N \rho_{s c}\left(E^{\prime}\right)}\right)\right] \\
& =0
\end{aligned}
$$

i.e. the appropriately normalized correlation functions of the measure $\mu_{\beta, V}^{(N)}$ at the level $E$ in the bulk of the limiting density asymptotically coincide with those of the Gaussian case and they are independent of the value of $E$ in the bulk.

The earlier work on the universality of random matrices has been focused on invariant ensembles. Important progress was made since the late 1990's by FokasIts-Kitaev [21], Bleher-Its [3], Deift et al. [7, 10, 11], PasturShcherbina [29, 30] and more recently by Lubinsky [25]. These results concern the simpler $\beta=2$ case. For $\beta=1,4$, the universality was established only quite recently for analytic $V$ with additional assumptions [8, 9, 24, 31] using earlier ideas of Widom [34]. The final outcome of these analyses is that universality holds for the measure (7) in the sense that the short scale behavior of the correlation functions is independent of the potential $V$ (with appropriate assumptions) provided that $\beta$ is one of the classical values, i.e., $\beta \in\{1,2,4\}$, that corresponds to an underlying matrix ensemble. Previous results on the universality of the local statistics for Wigner matrices were restricted to Hermitian Wigner matrices with a substantial Gaussian component, i.e., matrix of the form $H_{t}=e^{-t / 2} H_{0}+$ $\sqrt{1-e^{-t}} U$ where $H_{0}$ is a Wigner matrix and $U$ is an independent GUE matrix. The key element to analyze this ensemble is the Harish-Chandra-Itzykson-Zuber formula.
It was first put into a mathematically useful form by Johansson [23] (see also the later work of Ben Arous-Péche [1]) to prove the universality of Gaussian divisible ensembles with a Gaussian component of size order one. In [15], the size of the Gaussian component needed for proving the universality was greatly reduced to $N^{-1 / 2+\varepsilon}$. More importantly, the idea of approximating Wigner ensembles by Gaussian divisible ones was first introduced and this resulted in the first proof of universality for Hermitian ensembles with general smooth distributions for matrix elements. The smoothness condition was later on removed in $[33,16]$ for the Hermitian case. The symmetric ensembles with mild regularity conditions on the distributions of matrix elements were solved in [17], where the Dyson Brownian idea was introduced. The regularity assumptions were removed in [19].

\section{References}

[1] Ben Arous, G., Péché, S.: Universality of local eigenvalue statistics for some sample covariance matrices. Comm. Pure Appl. Math. LVIII (2005), 1-42.

[2] Berry, M.V., Tabor, M.: Level clustering in the regular spectrum, Proc. Roy. Soc. A 356 (1977), 375-394.

[3] Bleher, P., Its, A.: Semiclassical asymptotics of orthogonal polynomials, Riemann-Hilbert problem, and universality in the matrix model. Ann. of Math. 150 (1999), 185-266.

[4] Bohigas, O.; Giannoni, M.-J.; Schmit, C.: Characterization of chaotic quantum spectra and universality of level fluctuation laws. Phys. Rev. Lett. 52 (1984), no. 1, 1-4.

[5] Bourgade, P., Erdős, Yau, H.-T.: Universality of General $\beta$-Ensembles. arXiv:1104.2272.

[6] Bourgade, P., Erdős, Yau, H.-T.: Bulk Universality of General $\beta$-Ensembles with Non-convex Potential. arXiv:1201.2283.

[7] Deift, P.: Orthogonal polynomials and random matrices: a Riemann-Hilbert approach. Courant Lecture Notes in Mathematics 3, American Mathematical Society, Providence, RI, 1999.

[8] Deift, P., Gioev, D.: Universality in random matrix theory for orthogonal and symplectic ensembles. Int. Math. Res. Pap. IMRP 2007, no. 2, Art. ID rpm004, 116 pp.

[9] Deift, P., Gioev, D.: Random Matrix Theory: Invariant Ensembles and Universality. Courant Lecture Notes in Mathematics 18, American Mathematical Society, Providence, RI, 2009.

[10] Deift, P., Kriecherbauer, T., McLaughlin, K.T-R, Venakides, S., Zhou, X.: Uniform asymptotics for polynomials orthogonal with respect to varying exponential weights and applications to universality questions in random matrix theory. Comm. Pure Appl. Math. 52 (1999), 1335-1425.

[11] Deift, P., Kriecherbauer, T., McLaughlin, K.T-R, Venakides, S., Zhou, X.: Strong asymptotics of orthogonal polynomials with respect to exponential weights. Comm. Pure Appl. Math. 52 (1999), 1491-1552.

[12] Dyson, F.J.: Statistical theory of energy levels of complex systems, I, II, and III. J. Math. Phys. 3 (1962), 140-156, 157-165, 166-175.

[13] Dyson, F.J.: Correlations between eigenvalues of a random matrix. Commun. Math. Phys. 19 (1970), 235-250.

[14] Erdős, L., Knowles, A., Yau, H.-T., Yin, J.: Spectral Statistics of Erdős-Rényi Graphs II: Eigenvalue Spacing and the Extreme Eigenvalues. Preprint arXiv:1103.3869.

[15] Erdős, L., Péché, G., Ramírez, J., Schlein, B., and Yau, H.-T., Bulk universality for Wigner matrices. Commun. Pure Appl. Math. 63 (2010), no. 7, 895-925.

[16] Erdős, L., Ramirez, J., Schlein, B., Tao, T., Vu, V., Yau, H.-T.: Bulk Universality for Wigner Hermitian matrices with subexponential decay. Math. Res. Lett. 17 (2010), no. 4, 667-674. 
[17] Erdős, L., Schlein, B., Yau, H.-T.: Universality of random matrices and local relaxation flow. Invent. Math. 185 (2011), no. 1, 75-119.

[18] Erdős, L., Yau, H.-T., Yin, J.: Bulk universality for generalized Wigner matrices. Preprint arXiv:1001.3453.

[19] Erdős, L., Yau, H.-T., Yin, J.: Universality for generalized Wigner matrices with Bernoulli distribution. J. of Combinatorics, 1 (2011), no. 2, 15-85.

[20] Erdős, L., Yau, H.-T., Yin, J.: Rigidity of Eigenvalues of Generalized Wigner Matrices. Preprint arXiv:1007.4652.

[21] Fokas, A. S., Its, A. R., Kitaev, A. V.: The isomonodromy approach to matrix models in 2D quantum gravity. Comm. Math. Phys. 147 (1992), 395-430.

[22] Gaudin, M.: Sur la loi limit de l'espacement des valeurs propres d'une matrice aléatoire. Nucl. Phys. 25, 447-458.

[23] Johansson, K.: Universality of the local spacing distribution in certain ensembles of Hermitian Wigner matrices. Comm. Math. Phys. 215 (2001), no. 3, 683-705.

[24] Kriecherbauer, T., Shcherbina, M.: Fluctuations of eigenvalues of matrix models and their applications. Preprint arXiv:1003.6121.

[25] Lubinsky, D.S.: A New Approach to Universality Limits Involving Orthogonal Polynomials. Annals of Mathematics 170 (2009), 915-939.

[26] Mehta, M.L.: Random Matrices. Third Edition, Academic Press, New York, 1991.
[27] Mehta, M.L.: A note on correlations between eigenvalues of a random matrix. Commun. Math. Phys. 20 (1971), no. 3, 245-250.

[28] Mehta, M.L., Gaudin, M.: On the density of eigenvalues of a random matrix. Nuclear Phys. 18 (1960), 420-427.

[29] Pastur, L., Shcherbina, M.: Universality of the local eigenvalue statistics for a class of unitary invariant random matrix ensembles. J. Stat. Phys. 86 (1997), 109-147.

[30] Pastur, L., Shcherbina M.: Bulk universality and related properties of Hermitian matrix models. J. Stat. Phys. 130 (2008), no. 2, 205-250.

[31] Shcherbina, M.: Orthogonal and symplectic matrix models: universality and other properties. Preprint arXiv:1004.2765.

[32] Spencer, T.: Review article on random band matrices. Draft in preparation.

[33] Tao, T. and Vu, V.: Random matrices: Universality of the local eigenvalue statistics. Acta Math. 206 (2011), no. 1, 127-204.

[34] Widom H.: On the relation between orthogonal, symplectic and unitary matrix ensembles. J. Statist. Phys. 94 (1999), no. 3-4, 347-363.

[35] Wigner, E.: Characteristic vectors of bordered matrices with infinite dimensions. Ann. of Math. 62 (1955), 548-564.

[36] Wishart, J.: The generalized product moment distribution in samples from a Normal multivariate population. Biometrika 20A (1928), 32-52. 